\title{
QUALIDADE HIGIÊNICO-SANITÁRIA DA TILÁPIA (Oreochromis spp.) FRESCA E CONGELADA EM MERCADOS PÚBLICOS
}

\section{SANITARY-HYGIENIC QUALITY OF FRESH AND FROZEN TILAPIA (Oreochromis spp.) IN PUBLIC MARKETS}

\author{
Renata Xavier Silva ${ }^{1}$ \\ Maria Rociene Abrantes ${ }^{1 *}$ \\ Jeane Paiva Acioli Nascimento ${ }^{1}$ \\ Carolina de Gouveia M. E. Pinheiro ${ }^{1}$ \\ César Leandro Pereira Filgueira ${ }^{1}$ \\ Jean Berg Alves Silva ${ }^{1}$ \\ ${ }^{1}$ Universidade Federal Rural do Semi-Árido, Mossoró, RN, Brasil \\ *Autora para correspondência - rocienevet3@hotmail.com
}

\section{Resumo}

Objetivou-se verificar a qualidade higiênico-sanitária da tilápia (Oreochromis spp.), comercializada em mercados públicos do município de Mossoró-RN. Para isso, foi realizado, através de checklist, um estudo observacional, para avaliação das boas práticas de manipulação em 10 estabelecimentos de comercialização do peixe. Foram obtidas 30 amostras de tilápia avaliadas quanto aos aspectos sensoriais, microbiológicos (Salmonella spp., coliformes a $45{ }^{\circ} \mathrm{C}$, contagem de Staphylococcus ssp., bactérias psicrotróficas e enterobactérias) e quanto ao $\mathrm{pH}$ e temperatura interna e externa. Observou-se que nenhum dos estabelecimentos apresentou conformidade com os padrões recomendados pela legislação. Já em relação à avaliação sensorial, 83,3\% das amostras analisadas apresentavam conformidade com as exigências da legislação. Quanto à pesquisa de coliformes a $45^{\circ} \mathrm{C}$, os resultados variaram de 0,477 a $1,509 \log 10 \mathrm{UFC} / \mathrm{g}$, na pesquisa de Staphylococcus spp., de 4,854 a 5,397 $\log 10 \mathrm{UFC} / \mathrm{g}$ e ainda 3,884 a 4,937 e 4,707 a 5,463 log $10 \mathrm{UFC} / \mathrm{g}$ para enterobactérias e psicrotróficas, respectivamente. As amostras apresentaram-se dentro do exigido para a contaminação por Salmonella spp.(ausência). O $\mathrm{pH}$ variou de 5,99 a 6,18 e as temperaturas indicaram desconformidade com o recomendado. Assim, a qualidade higiênico-sanitária da tilápia comercializada em mercados públicos de Mossoró-RN estava comprometida, apresentando risco à saúde do consumidor pelas condições insatisfatórias.

Palavras-chave: boas práticas; físico-química; microbiologia.

\begin{abstract}
This study aimed to verify the sanitary quality of tilapia (Oreochromis spp.) sold in public markets in the city of Mossoro-RN. For such we made use of a checklist and an observational study to evaluate good handling practices in 10 establishments that commercialize fish. We obtained 30 samples of tilapia and evaluated them as to their sensory and microbiological aspects (Salmonella spp., coliforms at $45^{\circ} \mathrm{C}$, Staphylococcus ssp. count, psychrotrophic bacteria and enterobacteria), as well as to their $\mathrm{pH}$ and internal and external temperature. We observed that none of the studied establishments were in accordance with the standards recommended by the legislation. Regarding the sensory evaluation, $83.3 \%$ of the samples showed compliance with the requirements of the legislation. As for the coliforms at $45{ }^{\circ} \mathrm{C}$ essay, the results ranged from 0.477 to $1.509 \log 10 \mathrm{CFU} / \mathrm{g}$; in the Staphylococcus spp. essay we found results from 4.854 to $5.397 \log 10 \mathrm{CFU} / \mathrm{g}$; and further, we found values from 3.884 to 4.937 and from 4.707 to 5.463 $\log 10 \mathrm{CFU} / \mathrm{g}$ for enterobacteria and psychrotrophic bacteria, respectively. All the samples were within the requirements for contamination by Salmonella spp. (absence). The $\mathrm{pH}$ ranged from 5.99 to 6.18 and temperatures indicated non-compliance with the recommendations. Thus, we can infer that the sanitary quality of tilapia sold in public markets in Mossoro-RN was impaired, presenting a risk to consumer health due to unsatisfactory handling conditions.
\end{abstract}


Keywords: best practices; microbiology; physio-chemistry.

Enviado em: 04 agosto de 2016

Aceito em: 22 de agosto de 2016

\section{Introdução}

O pescado faz parte da dieta alimentar do ser humano e vem ganhando destaque cada vez mais, por apresentar fácil digestibilidade e elevados teores de proteínas, vitaminas e sais minerais ${ }^{(1)}$. A Organização Mundial da Saúde (OMS) recomenda o consumo médio de $12 \mathrm{~kg}$ de pescado ou derivados por pessoa por ano. No Brasil, a média de consumo é de apenas $9 \mathrm{~kg}$ por habitante.

Esse alimento é considerado como um produto altamente perecível e pode passar por processos de deterioração quando estocado, processado ou distribuído inadequadamente, levando ao crescimento microbiano, tornando-o inseguro para o consumidor $^{(2)}$. Fatores como contaminação do ambiente, condições higiênico-sanitárias durante a captura e manuseio, armazenamento, transporte e acondicionamento inadequados favorecem a degradação do alimento ${ }^{(1)}$.

Alimentos crus, comercializados em feiras livres e mercados públicos podem ser veículos de contaminação de microrganismos causadores de toxinfecção, colocando em risco a saúde do consumidor ${ }^{(3)}$.

É fundamental que o consumidor exija um pescado de qualidade, que satisfaça suas necessidades naturais e que seja um alimento seguro para o consumo. Assim, considerando a importância de se conhecer os possíveis riscos de contaminação microbiológica deste produto durante a comercialização, o presente trabalho teve por objetivo, avaliar a qualidade higiênico-sanitária da tilápia comercializada em feiras livres e mercados públicos na cidade de Mossoró-RN.

\section{Materiais e Métodos}

A pesquisa foi realizada em dez estabelecimentos (A - J), cinco em feiras livres e cinco em mercados públicos no município de Mossoró-RN. Foram realizadas três coletas, totalizando trinta amostras de peixe da espécie Oreochromis spp., popularmente conhecido como tilápia.

As amostras foram adquiridas inteiras, mantidas dentro da própria embalagem, como recomenda a RDC $\mathrm{n}^{\mathrm{o}} 12,02$ de janeiro de $2001^{(4)}$, e armazenadas dentro de uma caixa isotérmica com gelo eutético para serem transportadas até o Laboratório de Inspeção de Produtos de Origem Animal - LIPOA, na Universidade Federal Rural do Semiárido (UFERSA).

Como forma de padronizar as observações realizadas nos estabelecimentos, foi elaborado um checklist, segundo as normas estabelecidas pelo RDC ${ }^{0} 216 / 04^{(5)}$ da ANVISA e pelo Decreto 6.2345/86. Os estabelecimentos foram classificados como conforme (C) e não conforme (NC). As observações foram realizadas no momento da obtenção das amostras por meio de inspeção visual no ambiente, equipamentos e manipulação do pescado.

Após a aquisição das amostras coletadas, foi realizada avaliação organoléptica, segundo os critérios adotados pelo Regulamento da Inspeção Industrial e Sanitária de Produtos de Origem Animal (RIISPOA) (6).

Os dados foram coletados levando-se em consideração as seguintes características; aparência da pele (brilhante, úmida e sem laceração); escamas unidas, fortemente aderidas à pele, translúcidas e com brilho; guelras róseas, úmidas, brilhantes, ausência ou discreta presença de muco translúcido; opérculo rígido, resistência a abertura; olhos salientes, transparentes e brilhantes; carne firme, elástica e aderida aos ossos, além do odor suave ou ausente.

Para análise microbiológica foram pesadas $25 \pm 0,2 \mathrm{~g}$ da amostra, adicionado a $225 \mathrm{~mL}$ de água 
peptonada $0,1 \%$, correspondendo à primeira diluição $\left(10^{-1}\right)$, homogeneizada por aproximadamente dois minutos em "stomacher" a uma rotação média de $250 \mathrm{rpm}$. Feito isso, mais duas diluições sucessivas em tubos de ensaio contendo $9 \mathrm{~mL}$ de água peptonada $0,1 \%$ deram sequência à preparação das amostras. A qualidade microbiológica foi realizada pela pesquisa de Salmonella spp., quantificação de Staphylococcus spp., enterobactérias, bactérias psicrotróficas e enumeração de coliformes a $45^{\circ} \mathrm{C}$ de acordo com as recomendações da Instrução Normativa $n^{\circ}$ 62, de 26 de agosto de 2003, do Ministério da Agricultura, Pecuária e Abastecimento ${ }^{(7)}$. Para a determinação do $\mathrm{pH}$ (potencial hidrogeniônico) foi utilizado o equipamento da marca Hanna ${ }^{\circledR}$, de código - HI 99163 pH/temperature meter.

A temperatura do peixe foi aferida após a aquisição das amostras. A temperatura interna foi verificada com o auxílio do Digital Thermometer - food thermometer ${ }^{\circledR}$, introduzido na musculatura do pescado, e a temperatura externa com Infrared DT8380 Thermometer ${ }^{\circledR}\left(-50^{\circ} \mathrm{C}\right.$ a $\left.380^{\circ} \mathrm{C}\right)$.

\section{Resultados e Discussão}

Após a análise dos dados dos estabelecimentos, contidos no checklist, eles foram classificados e tabelados de acordo com a conformidade e não conformidade recomendada pela RDC $\mathrm{n}^{0}$ 216/04 da ANVISA ${ }^{(5)} \mathrm{e}$ pelo Decreto $n^{\circ} 6.2345 / 86$ (Tabela 1 ).

Tabela 1. Percentual de conformidade e não conformidade dos itens avaliados na comercialização da tilápia em Mossoró-RN, 2013

\begin{tabular}{llll}
\hline Checklist & & & \\
& Conforme & $\begin{array}{l}\text { Não } \\
\text { conforme }\end{array}$ & $\begin{array}{l}\text { Não } \\
\text { observado }\end{array}$ \\
\hline AMBIENTE & & & \\
\hline Encontra-se limpo? & $0 \%$ & $100 \%$ & - \\
Presença de animais ou insetos? & $0 \%$ & $100 \%$ & - \\
Lixo longe do manuseio? & $0 \%$ & $63,3 \%$ & $36,7 \%$ \\
1234567lo Lixeira fechada? & $0 \%$ & $100 \%$ & - \\
Lixeira com pedal? & $0 \%$ & $100 \%$ & - \\
\hline MANIPULADORES & & & - \\
\hline Tem adornos? & $50 \%$ & $50 \%$ & - \\
Uso de EPI's? & $13,4 \%$ & $86,6 \%$ & - \\
EPI's apresentam-se em estado adequado? & $16,6 \%$ & $83,3 \%$ & - \\
A roupa é de cor clara (branca)? & $40 \%$ & $60 \%$ & - \\
As unhas estão limpas? & $13,4 \%$ & $86,6 \%$ & - \\
Lavagem das mãos antes da manipulação? & $13,4 \%$ & $86,6 \%$ & - \\
\hline UTENSÍLIOS & & & - \\
\hline São higienizados antes de usar? & $6,6 \%$ & $80 \%$ & $13,3 \%$ \\
Apresenta-se descascado, enferrujado? & $65,3 \%$ & $34,6 \%$ & - \\
Está longe de contaminação? & $0 \%$ & $100 \%$ & - \\
\hline CONSERVAÇÕES DO PEIXE & & & - \\
\hline Exposição adequada do peixe? & $50 \%$ & $50 \%$ & - \\
Peixe sobre o gelo? & $50 \%$ & $50 \%$ & - \\
\hline
\end{tabular}

Modelo baseado na Resolução 216/2004 MS/ANVISA 
Pode-se observar que, em relação ao ambiente, $100 \%$ dos pontos de comercialização não se encontravam limpos, havia presença de insetos e animais e as lixeiras presentes estavam abertas e não apresentavam pedal. Quanto aos manipuladores nenhum estabelecimento apresentou 100\% de conformidade, demostrando ausência ou práticas inadequadas de Boas Práticas. Da mesma forma, os utensílios encontravam-se em desconformidade em todos os estabelecimentos, principalmente quanto à proximidade de fontes de contaminação, como cestos de lixo. Já o que se refere à conservação do pescado, $50 \%$ dos estabelecimentos mantinham o alimento em exposição adequada e sobre o gelo em escamas. Dos estabelecimentos que não apresentavam a exposição adequada, o pescado era mantido sobre balcões de madeira ou de alvenaria revestido com azulejo em condições inadequadas, propiciando à contaminação do produto. De acordo com a legislação vigente, é proibido manter o pescado fora de conservação frigorífica, exceto durante a fase de limpeza e evisceração.

As amostras foram avaliadas quanto as características sensoriais de acordo com as exigências adotadas pelo Regulamento de Inspeção Industrial e Sanitária de Produtos de Origem Animal (RIISPOA) ${ }^{(6)}$ (Tabela 2).

Tabela 2. Percentual das características sensoriais, conformes e não conformes, em tilápia (Oreochromis spp) comercializada em Mossoró-RN, 2013

Características sensoriais

\begin{tabular}{|c|c|c|}
\hline & Conforme & $\begin{array}{l}\text { Não } \\
\text { conforme }\end{array}$ \\
\hline Aparência da pele (brilhante, úmida e sem laceração) & $96,6 \%$ & $3,4 \%$ \\
\hline Escamas unidas fortemente aderidas à pele, translúcidas e com brilho & $93,3 \%$ & $6,7 \%$ \\
\hline $\begin{array}{l}\text { Guelras róseas, úmidas, brilhantes, ausência ou discreta presença de } \\
\text { muco translúcido }\end{array}$ & $93,3 \%$ & $6,7 \%$ \\
\hline Opérculo rígido, resistência à abertura & $100 \%$ & $0 \%$ \\
\hline Olhos salientes, transparentes e brilhantes & $86,6 \%$ & $13,4 \%$ \\
\hline Carne firme, elástica e aderida aos ossos & $90 \%$ & $10 \%$ \\
\hline Odor suave ou ausente & $90 \%$ & $10 \%$ \\
\hline
\end{tabular}

Pode-se observar na Tabela 2 que, das 30 amostras analisadas, 25 delas estavam conforme os parâmetros desejados pelo RIISPOA, totalizando $83,3 \%$. As outras cinco amostras, 16,7\%, apresentavam pelo menos um parâmetro em não conformidade com a legislação ${ }^{(6)}$.

De acordo com os resultados obtidos pela análise sensorial, a maioria das amostras estava apta para o consumo e comercialização. No entanto, as falhas na manipulação, conservação e/ou exposição do pescado (Tabela 1) contribuem para a perda da qualidade do pescado e início de deterioração.

Não foi detectada a presença de Salmonella spp. nas amostras analisadas, atendendo, portanto, ao padrão de ausência desse microrganismo em $25 \mathrm{~g}$ do pescado, estabelecido pelo RDC $\mathrm{n}^{\circ} 12,02$ de janeiro de $2001^{(4)}$. Esses resultados diferiram dos de Duarte et al. ${ }^{(1)}$, que encontraram presença de Salmonella spp. nos pescados analisados no Nordeste brasileiro.

Os resultados referentes às médias e desvio-padrão para a pesquisa de Staphylococcus spp., bactérias psicrotróficas, coliformes a $45^{\circ} \mathrm{C}$ e enterobactérias das amostras analisadas encontram-se na Tabela 3.

Observou-se contaminação das amostras de tilápias neste trabalho para Staphylococcus spp. com valor acima de 4,854 $\log 10 \mathrm{UFC} / \mathrm{g}$, superior ao padrão estabelecido pelo RDC $\mathrm{n}^{\mathrm{o}} 12^{(4)}$ para estafilococos coagulase positiva, máximo de $10^{3} \mathrm{UFC} / \mathrm{g}$, em $\log 10$ corresponde à $3 \mathrm{UFC} / \mathrm{g}$. 
Tabela 3. Valores das médias em logaritmo e desvio padrão das contagens de Staphylococcus spp., bactérias psicrotróficas, coliformes a $45^{\circ} \mathrm{C}$ e enterobactérias das amostras de tilápia (Oreochromis spp) em Mossoró-RN, 2013

\begin{tabular}{lcccc}
\hline Estabelecimentos & $\begin{array}{c}\text { Staphylococcus } \\
\text { spp. } \\
\text { (UFC/g) }\end{array}$ & $\begin{array}{c}\text { Bactérias } \\
\text { psicrotróficas } \\
\text { (UFC/g) }\end{array}$ & $\begin{array}{c}\text { Coliformes a } \\
\mathbf{4 5}^{\circ} \mathbf{C} \\
(\mathbf{N M P} / \mathbf{g})\end{array}$ & $\begin{array}{c}\text { Enterobactérias } \\
\text { (UFC/g) }\end{array}$ \\
\hline A & & & & \\
B & $5,397 \pm 0$ & $5,410 \pm 0,189$ & $1,509 \pm 0,807$ & $4,711 \pm 0,050$ \\
C & $5,397 \pm 0$ & $5,301 \pm 0$ & $1,509 \pm 0,744$ & $3,943 \pm 0,403$ \\
D & $5,247 \pm 0,211$ & $5,251 \pm 0,085$ & $0,639 \pm 0,280$ & $4,890 \pm 0,189$ \\
E & $5,284 \pm 0,195$ & $4,926 \pm 0,674$ & $0,503 \pm 0,045$ & $4,727 \pm 0,247$ \\
F & $5,260 \pm 0,237$ & $5,463 \pm 0,423$ & $0,974 \pm 0,861$ & $3,884 \pm 0,505$ \\
G & $4,854 \pm 0,643$ & $5,278 \pm 0,489$ & $0,503 \pm 0,045$ & $4,100 \pm 1,014$ \\
H & $5,153 \pm 0,304$ & $5,387 \pm 0,131$ & $0,800 \pm 0,560$ & $4,937 \pm 0,108$ \\
I & $5,397 \pm 0$ & $4,805 \pm 0,355$ & $0,579 \pm 0,177$ & $4,492 \pm 0,636$ \\
J & $5,366 \pm 0,054$ & $4,707 \pm 0,504$ & $0,477 \pm 0$ & $4,266 \pm 0,762$ \\
\hline
\end{tabular}

A ocorrência de manipulação incorreta do alimento pode ocasionar contaminação por Staphylococcus spp. pois o manipulador é o principal veiculador destes micro-organismos, que se alojam preferencialmente nas fossas nasais, boca e pele ${ }^{(8)}$. A ineficiência dos métodos de manipulação e higiene empregados favorecem a instalação e crescimento deste micro-organismo patogênico. Diferindo do presente trabalho, Soares et al. ${ }^{(9)}$ não verificaram Staphylococccus aureus em tilápia do Nilo (Oreochromis niloticus) no município de Apodi-RN.

Com relação à contagem de bactérias psicotróficas os resultados variaram de 4,707 a 5,463 $\log 10 \mathrm{UFC} / \mathrm{g}$ (Tabela 3 ) e foram analisados como forma de auxiliar a pesquisa em relação à qualidade microbiológica. A legislação brasileira não estabelece limites máximos toleráveis para esse grupo de micro-organismos. Portanto, foram utilizados os valores estabelecidos pela International Comission Microbiological Specications for Foods (ICMSF) ${ }^{(10)}$, de 7,0 log $10 \mathrm{UFC/g}$ de amostra de bactérias psicrotróficas. Deste modo, constatou-se que nenhum dos estabelecimentos apresentou média superior aos valores estabelecidos pela ICMSF.

Em relação à pesquisa para enterobactérias, os resultados variaram de 3,884 a 4,937 log10UFC/g. Não há dados na legislação para a verificação de enterobactérias em peixe, mas considerando-se os valores das médias e também das amostras que obtiveram elevado crescimento de colônias, as mesmas podem oferecer riscos à saúde do consumidor. A presença dessas bactérias é preocupante por serem os principais agentes de infecções intestinais ${ }^{(11)}$. De acordo com Baudart et al. ${ }^{(12)}$, a contaminação de Enterobacteriaceae pode causar graves doenças transmitidas por alimentos.

Em alguns dos estabelecimentos visitados (A, B e C), o pescado estava exposto ao ar livre, sem nenhum tipo de conservação ou refrigeração (gelo, caixa térmica ou bancada de inox), promovendo a elevação da temperatura externa e interna, facilitando o processo de degradação, além de que o descongelamento não uniforme proporciona perda da qualidade.

Em relação ao pH das amostras analisadas, todas apresentavam-se dentro dos padrões estabelecidos pela Legislação Federal, com o pH dentro dos limites de 5,4 a 6,5(6). Vale ressaltar a importância de 
se avaliar o pH para se verificar a qualidade do pescado, porém esse não deve ser o único parâmetro para determinar o índice de frescor.

O pescado era vendido como sendo peixe fresco ou congelado, mas, de acordo com os dados coletados das temperaturas externa e interna dos mesmos, pôde-se perceber que eles não estavam dentro dos padrões estabelecidos pela legislação. Para ser considerado fresco o pescado não deverá ter sofrido qualquer processo de conservação, a não ser do gelo ${ }^{(6)}$. Das 30 amostras, 14 foram coletadas como sendo peixe fresco $(46,6 \%)$ e 16 amostras, como congelado $(53,4 \%)$, nas quais as temperaturas externas variaram de $1,4{ }^{\circ} \mathrm{C}$ a $15,84{ }^{\circ} \mathrm{C}$ e as temperaturas internas variaram de $9{ }^{\circ} \mathrm{C} \mathrm{a} 18,66{ }^{\circ} \mathrm{C}$.

Araújo et al. ${ }^{(13)}$, na sua avaliação da qualidade da tilápia (Oreochromis spp.) comercializada em feiras livres do município de Mossoró - RN, demonstraram que das 36 amostras de tilápias analisadas, 69\% apresentaram-se fora dos padrões estabelecidos pela legislação.

\section{Conclusão}

Embora as condições higiênicas, sanitárias e as condições de comercialização da tilápia comercializada em feiras livres e mercado público de Mossoró-RN estivessem insatisfatórias, as características sensoriais e microbiológicos das amostras analisadas estavam adequadas para o consumo.

\section{Referências}

1 Duarte DAM, Ribeiro AR, Vasconcelos AMM, Silva JVD, Andrade PLA, Santana AAP. Ocorrência de Salmonella spp. e Staphylococcus coagulase positiva em pescado no nordeste, Brasil. Arquivo do Instituto Biológico. 2010; 77(4):711-713.

2 Ferreira EM, Lopes IS, Pereira LCR, Costa FN. Qualidade microbiológica do peixe serra (Scomberomerus brasiliensis) e do gelo utilizado na sua conservação. Arquivo do Instituto Biológico. 2014; 81(1):49-54.

3 Correia M, Roncada MJ. Características microscópicas de queijos prato, mussarela e mineiro comercializados em feiras livres da Cidade de São Paulo. Revista de Saúde Pública. 1997; 3(31):296-301.

4 Brasil. Ministério da saúde. Agência Nacional de Vigilância Sanitária. Resolução RDC nº 12, de 02/01/2001. Regulamento Técnico Sobre os Padrões Microbiológicos para Alimentos. Diário Oficial da União. Brasília: 2001.

5 Brasil. Ministério da saúde. Agência Nacional de Vigilância Sanitária. Resolução RDC n.216, de 15\09\2004. Regulamento Técnico de Boas Práticas para Serviços de Alimentação Brasília, Diário Oficial da União, Brasília:2004.

6 Brasil. Ministério da Agricultura, Pecuária e Abastecimento. Decreto n 30.691, de 29 de março de 1952. Aprova o novo Regulamento de Inspeção Industrial e Sanitária de Produtos de Origem Animal (RIISPOA). Diário Oficial da União. Brasília, DF, 7 jul. 1952. Seção 1, p. 10785.

7 Brasil. Ministério da Agricultura. Instrução Normativa n 62 de 26 de Agosto de 2003. Oficializa os Métodos Analíticos Oficiais para Análises Microbiológicas para Controle de Produtos de Origem Animal e Água. Diário Oficial da União, Brasília, 2003.

8 Ribeiro ALMS, Oliveira GM, Ferreira VM, Pereira MMD, Silva PPO. Avaliação microbiológica da qualidade do pescado processado, importado no estado do Rio de Janeiro. Revista brasileira de Ciência Veterinária. 2009; 16(3):109-112.

9 Soares VM, Pereira JG, Izidoro TB, Martins AO, Pinto JPAN, Biondi GF. Qualidade

Cienc. anim. bras., Goiânia, v.17, n.4, p. 574-580 out./dez. 2016 
Microbiológica de Filés de Peixe Congelados Distribuídos na cidade de Botucatu-SP. UNOPAR Científica Ciências Biológicas e da Saúde. 2011;13(2):85-8.

10 International Comission Microbiological Specications for Foods. (ICMSF). Disponível em $<$ http://www. icmsf.org/ > acesso em 06/09/2013.

11 Sousa CP. Segurança alimentar e doenças veiculadas por alimentos: utilização do grupo coliforme como um dos indicadores de qualidade de alimentos. Revista APS. 2006;9(1):83-88.

12 Baudart J, Robyns A, Peuchet S, Drocourt JL, Lebaron P. Sensitive counting of viable Enterobacteriaceae in seawaters and relationship with fecal indicators. Journal of Microbiological Methods. 2011;84:482-485.

13 Araújo DAFV, Soares KMp, Góis VA, Aroucha EMM, Silva JBA, Leite AI. Avaliação da qualidade da tilápia (Oreochromis sp) comercializada em feiras livres no município de Mossoró, RN. Higiene alimentar.2011;25(196/197):129-133. 